\title{
Importância do sistema nervoso no controle da micção e armazenamento urinário
}

\author{
The importance of the nervous system in the \\ control of voiding and urine storage
}

Rodrigo Ungari Juc ${ }^{1}$, Eduardo Colombari', Monica Akemi Sato ${ }^{1}$

\section{Resumo}

0 controle da micção, apesar da aparente simplicidade em seu funcionamento, apresenta vários níveis de regulação com relativa complexidade. Em estados conscientes e inconscientes, envolve a atividade de nervos periféricos, da medula sacral e das áreas centrais que constituem o bulbo, a ponte, o mesencéfalo e o córtex. Neste trabalho de revisão serão abordados quais são as áreas centrais atualmente conhecidas por participarem do controle da micção e armazenamento urinário, assim como a sinalização proveniente do sistema nervoso periférico, que se dirige ao trato urinário inferior. Realizouse uma busca nas bases de dados Pubmed e Scielo utilizando descritores para obter informações sobre o controle central da micção e 0 armazenamento urinário. A primeira porção do tronco encefálico que demonstrou relação com atividade miccional foi a ponte, principalmente o centro miccional pontino (PMC). É um ponto de convergência de estímulos pró e antimicção e sua principal função é ser o centro de comando para 0 início e harmonia do esvaziamento vesical. Diferentes regiões distintas disparam após a micção, sugerindo existir neurônios sinalizadores do início do relaxamento detrusor. A substância cinzenta periaquedutal é uma área importante no controle central do trato urinário inferior. Recebe modulação inibitória da região posterior hipotalâmica, envolvida no mecanismo de defesa (luta ou fuga). Outras estruturas como córtex, núcleos da base e cerebelo também vêm sendo evidenciadas por modular a miç̧ão. Embora neurônios bulbares sejam conhecidos por integrarem diferentes tipos de informações somatoviscerais e, principalmente, por participarem na regulação cardiovascular, pouco é conhecido sobre a importância desses neurônios sobre o controle vesical.

Palavras-chave: Bexiga urinária; controle; sistema nervoso simpático; sistema nervoso parassimpático.

\section{Abstract}

The micturition control, despite the apparent simplicity in its functioning, shows several levels of relative complexity. In conscious and unconscious states, it involves the activity of peripheral nerves, the sacral spinal cord, and the central areas that constitute the medulla oblongata, pons, midbrain, and cerebral cortex. In this review, the main currently known central areas involved in the urinary storage and voiding are focused, as well as the entrainment of peripheral signals related to the lower urinary tract. Pubmed and Scielo databases were searched using the keywords related to central control of voiding and urine storage. The first portion of the brainstem that demonstrated relationship to voiding activity was the pons, particularly the pontine micturition center (PMC). It is a point of convergence pro-stimuli and anti-urination and its main function is the command center for starting and harmonizing the bladder emptying. Different neurons of distinct regions fire after voiding, suggesting the existence of neurons signaling the initiation of detrusor relaxation. The periaqueductal gray matter is an important central area involved in the control of the lower urinary tract. It receives inhibitory modulation from posterior hypothalamic region, which is responsible for the defense mechanism (flight or fight). Other central structures such as the cerebral cortex, basal ganglia, and cerebellum have also been evidenced for modulating the micturition. Although medullary neurons are known for the integration of different somato-visceral signals and particularly for taking part in cardiovascular regulation, little is still known about the importance of those neurons in micturition control.

Keywords: Urinary bladder; control; sympathetic nervous system; parasympathetic nervous system.

Recebido: 30/4/2010

Revisado: 9/9/2010

Aprovado: 9/12/2010

\footnotetext{
Trabalho realizado na Faculdade de Medicina do ABC (FMABC), Santo André (SP), Brasil

Disciplina de Fisiologia da FMABC, Santo André, (SP), Brasil

Endereço para correspondência: Monica Akemi Sato - Faculdade de Medicina do ABC - Av: Lauro Gomes, 2.000 - Vila Sacadura Cabral - CEP 09060-650 -

Santo André (SP) - E-mail: monica.akemi.sato@gmail.com
} 


\section{Introdução}

O mecanismo de continência urinária possui, apesar da aparente simplicidade em seu funcionamento, um complexo controle em vários níveis. Uma vez adquirida a capacidade de armazenar urina na infância, a decisão de eliminá-la envolve percepção de enchimento completo da bexiga; decisão de esvaziá-la sem completo enchimento e decisão social de quando é apropriado iniciar a micção. Este controle em estados conscientes e inconscientes envolve a atividade de nervos periféricos, medula sacral e de área centrais que envolvem parte do bulbo, ponte, mesencéfalo e córtex cerebral ${ }^{1}$.

Na última década, com o advento da utilização de técnicas de imagem funcional do cérebro, diferentes aspectos centrais sobre o funcionamento da bexiga humana foram descobertos, sendo Fukuyama et $a l^{2}$ os pioneiros nestes estudos ${ }^{2}$. Como consequência desses estudos, mostrou-se pela primeira vez a ativação de centros superiores durante a micção, evidenciando-se in loco em humanos o núcleo de Barrington ${ }^{3}$ (descrito em 1921).

Considerando-se que o controle miccional e de armazenamento urinário envolve uma interação complexa entre diversas áreas do sistema nervoso central, e sua sintonia e integridade são fundamentais para o adequado gerenciamento dessas funções. Nesta revisão será abordado quais são as áreas centrais atualmente conhecidas por participarem desse controle, bem como a sinalização dependente do sistema nervoso periférico proveniente e também dirigida ao trato urinário inferior. Estes dados foram obtidos a partir de uma busca nas bases de dados Pubmed e Scielo, utilizando-se os descritores "urinary bladder and central nervous system, micturation, urinary bladder and sympathetic e urinary bladder and parasympathetic".

\section{Áreas do sistema nervoso central e controle miccional}

Diferentes áreas pontinas e suprapontinas têm sido evidenciadas como sendo importantes para o controle da micção.

\section{Estruturas pontinas}

A região do encéfalo mais importante para o controle central da micção é o tronco encefálico, composto pelo bulbo, ponte e mesencéfalo. As microrregiões dentro do tronco que, por sua vez, destacam-se no controle da micção são o centro miccional pontino (PMC), o centro pontino da continência (PCC) e a substância cinzenta periaquedutal (PAG).

A primeira porção do tronco encefálico, em que se demonstrou em modelos animais a relação com a atividade miccional, foi a ponte, em particular, o PMC, também conhecido como núcleo de Barrington ${ }^{3}$. A estimulação deste centro resultou em relaxamento do esfíncter uretral e contração detrusora, enquanto lesões neste evoluem para abolição da micção e retenção urinária completa.
O PMC é um ponto de convergência de estímulos pró e antimicção. Sua principal função é ser o centro de comando para o início e harmonia do esvaziamento vesical. A associação de dificuldades na micção com tumores em fossa posterior, pequenas lesões pontinas ${ }^{4,5}$, gliomas ${ }^{6}$ em tronco encefálico e lesões vasculares, demonstrou a importância da integridade da ponte dorsolateral para a manutenção dessa função. Nos humanos, esta região encontra-se amplamente ativa em situações de bexiga repleta. Diferentemente dos humanos, o PMC possui localização medial em relação ao PCC em gatos, por este motivo, tal centro recebeu a denominação de região $\mathbf{M}^{7}$.

O PMC é caracterizado como núcleo pré-motor da micção por possuir múltiplas projeções diretas para a medula espinhal e, desse modo, é responsável por modular a atividade da bexiga. Alguns dos neurônios do PMC disparam o gatilho para iniciar a micção, outros para mantê-la, ${ }^{8,9}$, e não há evidências de neurônios atuando em ambas as situações. Duas vias distintas partem do PMC: uma via direta contendo o neurônio pré-ganglionar parassimpático, que é responsável por estimular diretamente o músculo detrusor; e outra via indireta, que passa pela formação reticular medial e permite o relaxamento esfincteriano ${ }^{1}$.

O PMC possui vários tipos de conexões aferentes e eferentes (Figura 1). Dentre as aferências, destacam-se: a região lateral da PAG envia fortes impulsos estimulatórios ao PMC na vigência de distensão detrusora $\mathrm{a}^{10}$; enquanto o tegumento pontomedular modula o PMC alterando o nível de sensibilidade de seus neurônios ${ }^{11}$.

Microrregiões próximas ao PMC continuam disparando durante o relaxamento vesical, imediatamente após a micção, o que sugere a existência de neurônios que sinalizam quando se deve finalizar o processo contrátil vesical e quando deve ser iniciado o processo de relaxamento detrusor. Eles pertencem ao centro pontino da continência também conhecida como região L.

As projeções desta área parecem ser restritas ao núcleo de Onuf, que contém neurônios motores do esfíncter uretral. Portanto, o PCC tem função limitada em finalizar a micção com disparos precedendo o fim da micção ${ }^{1}$.

Não há evidências de conexões entre o PMC e o PCC, o que sugere que estas áreas funcionem de modo independente ${ }^{12}$. O PCC, localizado caudoventrolateralmente ao PMC, portanto, é o responsável por parar a micção, excitar a musculatura pélvica e contrair o esfíncter uretral.

A PAG também representa uma importante área no controle central do trato urinário inferior. Recebe informações de aferentes vesicais, além de constituir um grande centro integrador de informações em virtude de sua extensa gama de conexões com diferentes estruturas, como a amígdala, o córtex orbital e pré-frontal, a área pré-óptica hipotalâmica, o núcleo do trato solitário (NTS) e o PMC.

As aferências sacrais terminam nas regiões centrais da PAG e suas eferências ao PMC saem lateralmente. No entanto, regiões dorsorrostrais e ventrocaudais, quando estimuladas, impedem a micção por inibirem o PMC. Não existem também descrições de conexões entre a PAG e o PCC.

A PAG recebe modulação da região posterior hipotalâmica, a qual está envolvida com o mecanismo de defesa (luta ou fuga - flight or fi$g h t)$ que inibe a PAG e que, por sua vez, levará a um novo estímulo inibitório ao PMC causando retenção urinária ${ }^{13}$. 
Estudos de imagem demonstraram ativação considerável da PAG em situações de dor e distensão vesical, o que reforça sua provável participação no processamento de informações sensitivas aferentes ${ }^{14-16}$.

A PAG juntamente com outras áreas como o núcleo pré-óptico hipotalâmico estão envolvidas na inibição do PMC via liberação de GABA ${ }^{17}$.

Outros centros pontinos parecem participar, independentes ao PMC, no processo de início da micção, entre eles estariam a área reticular gigantocelular e magnocelular. Ambos apresentam ativação (também via medula espinhal) pouco antes ou concomitante às contrações vesicais. Ainda permanece desconhecido se estes seriam mecanismos redundantes ou protetores, em caso de falha do PMC, bem como ainda permanece incerto como é organizada a circuitaria nos núcleos do tronco encefálico no controle da micção.

\section{Estruturas suprapontinas}

As regiões suprapontinas possuem função primordialmente moduladora às estruturas do tronco encefálico, no que diz respeito ao controle miccional e de inibição da micção.

O reflexo bulbo-espinal por si só poderia levar ao esvaziamento involuntário da bexiga, assim que essa atingisse um nível crítico. Por ser socialmente inaceitável, a inibição do esvaziamento provém de modulações dos centros suprapontinos.

A PAG parece ter importância chave no registro de sensações de enchimento vesical e ao promover a modulação sobre quando seria o momento ideal para esvaziá-la sob influência cortical. Durante o enchimento vesical, centros cerebrais superiores podem suprimir qualquer

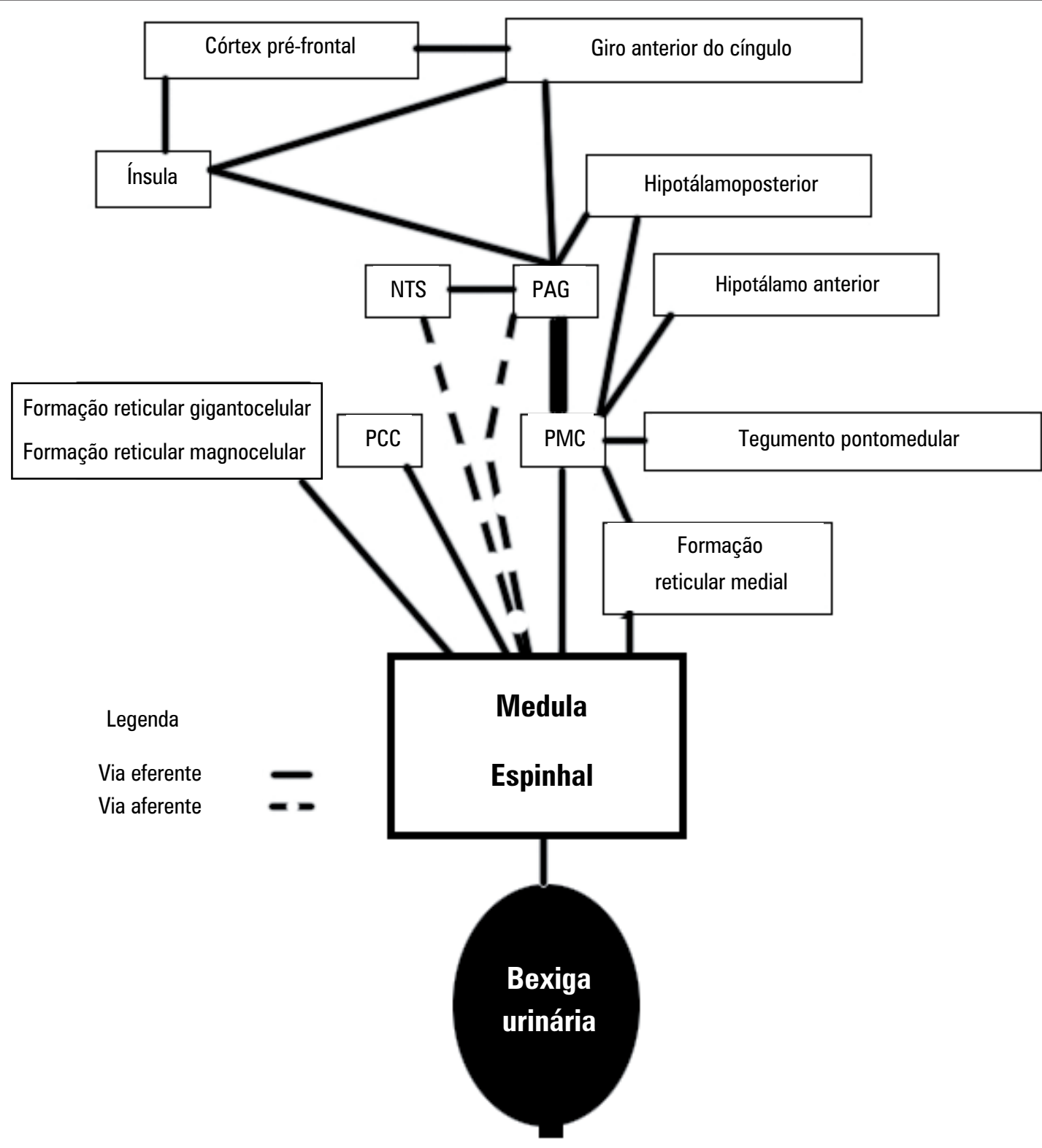

Figura 1 - Diagrama de possíveis conexões entre diversas estruturas cerebrais - centros superiores, tronco encefálico e trato urinário inferior. Linha contínua evidencia eferências e linha tracejada evidencia aferências. PMC: centro pontino da micção; PCC: centro pontino da continência; PAG: substância cinzenta periaquedutal; NTS: núcleo do trato solitário. 
estímulo excitatório da PAG ao PMC. Portanto, a excitação do PMC, via PAG, só é permitida em situações de segurança e aceitabilidade social ou quando tal modulação encontra-se ausente ou lesada.

A ínsula parece estar envolvida no processamento de informações aferentes vesicais. O desejo consciente de micção é perdido após lesões frontais, o que sugere que a integração do córtex frontal com a ínsula é essencial para a existência de tal sensação ${ }^{18}$.

O hipotálamo parece ter função inibitória sobre a PAG e, consequentemente, sobre o PMC, em situações não seguras. O hipotálamo anterior e caudal possui conexões monossinápticas com esses núcleos, e estudos recentes de imagem com cérebros humanos denotam atividades nestas áreas durante enchimento vesical ${ }^{19}$.

Estudos utilizando ressonância magnética mostraram que estruturas suprapontinas, como o córtex pré-motor medial, núcleos da base e cerebelo são ativadas por contração do assoalho pélvico de voluntá$\operatorname{rios}^{20}$. A hipótese levantada por este estudo era de que contrações da musculatura do assoalho pélvico resultariam em desejo de micção. A importância do cerebelo também foi evidenciada em pacientes com atrofia múltipla central, nos quais se observou diminuição da atividade do vermis cerebelar durante enchimento vesical ${ }^{21}$.

O giro anterior do cíngulo (GAC) também parece ser importante para o aparecimento de todas as etapas de funcionamento do trato urinário inferior: armazenamento, micção e interrupção voluntária miccional, visto que a incontinência era observada após infarto, pósacidente vascular cerebral isquêmico ou hemorrágico bilateral desta região encefálica. O GAC é crítico para o controle vesical e em pacientes portadores de urgeincontinência, esta área apresenta ativação exacerbada durante o enchimento vesical, mesmo na ausência de contrações detrusoras, sugerindo envolvimento tanto aferente quanto eferente ${ }^{18}$.

Outra evidência de que estruturas suprapontinas seriam relevantes no controle da micção deve-se à doença do lobo frontal ${ }^{22}$, descrita por Andrew e Nathan ${ }^{22}$. Essa doença apresenta característica peculiar: os atos miccionais ocorrem normalmente, todavia, a lesão do lobo frontal leva a frequentes episódios de urgências miccionais (às vezes, urgeincontinência), quando o paciente está acordado, e incontinência, quando dorme. Nenhum dos pacientes estudados apresentava demência ou era indiferente quanto aos seus sintomas. Ao contrário, mostravam-se extremamente insatisfeitos com suas condições e inclusive buscavam ajuda médica frequentemente.

Em 2005, Dasgupta et al. realizaram um estudo abordando pacientes hígidas em um grupo e pacientes portadoras de síndrome de Fowler, caracterizada por disfunção primária de relaxamento esfincteriano da bexiga, costumeiramente levando à retenção urinária aguda e alta pressão de repouso em jovens ${ }^{23}$. O grupo com a doença apresentou perda progressiva da sensação de enchimento e perda do desejo de urinar, no entanto, a simples estimulação elétrica dos nervos sacrais restaurou a sensibilidade e a habilidade miccional, apesar de a doença permanecer. A análise dos mecanismos centrais demonstrou que, no Grupo Controle, havia intensa atividade no mesencéfalo anterior e nas regiões ventrais e dorsais do giro do cíngulo. No grupo com síndrome de Fowler, com o enchimento vesical pleno, havia apenas estímulo em regiões difusas do córtex e, após terapia de eletroestimulação, normalizou-se a atividade no giro do cíngulo e, especialmente, no mesencéfalo. Portanto, sugeriuse uma função sensitiva do mesencéfalo na percepção de enchimento vesical. Os pacientes portadores de sintomas urinários com lesões em regiões corticais apresentam descontrole do trato urinário inferior, evidenciando a importância do córtex frontal sobre seu funcionamento.

Apesar de o sistema nervoso central conter importantes estruturas envolvidas no controle da micção, seu funcionamento é profundamente dependente de sinais periféricos que são provenientes e também são dirigidos ao trato urinário inferior.

\section{Sistema nervoso periférico: aferências, eferências e controle da micção}

A coordenação nervosa periférica da bexiga e esfíncter inferior externo ocorre por inervação parassimpática, simpática e somática, que emergem da região sacral e toracolombar da medula espinhal ${ }^{24-25}$.

Os nervos sacrais parassimpáticos (pélvicos) exercem ação excitatória (colinérgica e purinérgica) na bexiga e apresentam caráter inibitório na uretra. Os nervos hipogástricos representam a principal inervação simpática da bexiga ${ }^{24-26}$. A inervação simpática toracolombar excita (por liberação de noradrenalina) o esfíncter uretral, assim como inibe a atividade parassimpática e a contração das células musculares lisas do detrusor.

As mais importantes aferências para início da micção são provenientes dos nervos pélvicos, na região sacral. São compostos por fibras mielinizadas (A-delta) e não-mielinizadas (fibras C), as quais conduzem impulsos nervosos referentes à tensão, barorrecepção e nocirrecepção na parede vesical ${ }^{27}$.

Informações aferentes do trato urinário inferior ascendem ao sistema nervoso central em direção à PAG e ao NTS que, por sua vez, realizam modulações no hipotálamo e amígdala. Estas projeções, portanto, possibilitam a modulação hierarquizada dos reflexos somatossensoriais.

\section{Anatomia do trato urinário inferior}

O trato urinário é composto pelos rins, ureteres, bexiga urinária e uretra. Este se divide em superior (rins e ureteres) e inferior (bexiga e uretra).

\section{Bexiga urinária}

A bexiga urinária é uma víscera oca com paredes musculares fortes, caracterizada por sua grande distensibilidade. Trata-se de um reservatório temporário de urina, encontra-se na pelve menor (adultos) e é relativamente livre dentro do tecido gorduroso subcutâneo extraperitoneal, exceto pelo seu colo, que é mantido firmemente pelos ligamentos puboprostáticos, em homens, e pelos ligamentos pubovesicais, em mulheres. 
Este órgão pode ser dividido anatomicamente em ápice, corpo, fundo, colo e úvula.

O leito da bexiga é formado de cada lado pelos ossos do púbis e pelos músculos obturador interno e levantador do ânus e, posteriormente, pelo reto ou pela vagina. Sua parede é composta principalmente pelo músculo detrusor. Em direção ao colo da bexiga urinária masculina, as fibras musculares formam o músculo esfincteriano interno involuntário. Os óstios dos ureteres e o óstio interno da uretra estão no ângulo do trígono da bexiga. Os ureteres situam-se obliquamente em relação à parede da bexiga em uma direção ínfero-medial, o que, em situações de aumento de pressão intravesical, fisiologicamente comprime as paredes ureterais impedindo refluxo urinário.

O suprimento arterial vesical dá-se pelos ramos das artérias ilíacas internas. As artérias vesicais superiores suprem as partes antero superiores da bexiga e as artérias vesicais inferiores suprem o fundo e o colo. No entanto, nas mulheres, a nutrição do fundo e colo é realizada pelas artérias vaginais.

A drenagem venosa ocorre pelas tributárias das veias ilíacas internas. Em homens, o plexo venoso vesical combina-se com o plexo venoso prostático. O plexo venoso vesical drena principalmente por meio das veias vesicais inferiores para as veias ilíacas internas, no entanto, pode drenar para os plexos venosos vertebrais internos. Em mulheres, o plexo venoso vesical envolve a parte pélvica da uretra e o colo da bexiga urinária e comunica-se com o plexo venoso vaginal ou uterovaginal.

Em ambos os sexos, a drenagem linfática da face superior vesical dá-se para os linfonodos ilíacos externos e a face inferior e fundo dá-se para os linfonodos ilíacos internos. Alguns vasos provenientes do colo podem drenar para linfonodos sacrais ou ilíacos comuns.

A inervação da bexiga urinária é realizada por fibras parassimpáticas derivadas dos nervos esplâncnicos pélvicos, e as fibras simpáticas derivam-se dos nervos de T11 - L2. Esses formam o plexo nervoso vesical. As fibras parassimpáticas são motoras para o músculo detrusor e inibidoras para o músculo esfíncter interno. As fibras sensitivas provenientes da bexiga urinária são viscerais e seguem o trajeto parassimpático, como fazem aquelas que transmitem sensações de dor (distensão excessiva) provenientes da porção inferior vesical. Já as fibras de dor do teto da bexiga seguem as fibras simpáticas até gânglios espinais de T11 - L2.

\section{Uretra}

A uretra é um tubo muscular que conduz a urina do óstio interno da uretra da bexiga urinária até o óstio externo da uretra. A uretra masculina e a feminina apresentam particulares anatômicas importantes.

\section{Uretra masculina}

A uretra masculina divide-se em quatro porções: pré-prostática ou bulbar, prostática, membranosa e esponjosa. A porção prostática apresenta peculiaridades na face interna da parede posterior, cuja parte mais proeminente é a crista uretral com dois seios prostáticos (um de cada lado) onde os dúctulos prostáticos abrem-se. Na parte média da crista há o colículo seminal, uma eminência arredondada com uma fenda - utrículo prostático. Dentro do utrículo prostástico, por sua vez, há o ducto ejaculatório.

O suprimento arterial das duas primeiras porções proximais dá-se pelos ramos prostáticos das artérias vesicais inferiores e retais médias. As distais, pelas artérias que irrigam o pênis. Já a drenagem venosa segue as artérias e tem nomes semelhantes. A drenagem linfática segue principalmente para os linfonodos ilíacos externos.

A inervação uretral dá-se principalmente pelo plexo prostático e pelo nervo pudendo.

\section{Uretra feminina}

A uretra feminina de aproximadamente $4 \mathrm{~cm}$ de comprimento passa anteroinferiormente ao óstio interno da uretra da bexiga e o óstio externo da uretra encontra-se no vestíbulo da vagina logo anterior ao óstio da vagina. A uretra passa com a vagina por meio do diafragma da pelve, músculo esfíncter externo da uretra e membrana do períneo.

O suprimento arterial é dado pelas artérias pudenda interna e vaginal. A drenagem é dada pelas veias pudenda interna e vaginal. Os linfonodos sacrais e ilíacos internos são os que mais participam da drenagem linfática.

A inervação da uretra feminina assemelha-se com a masculina: originam primordialmente do nervo pudendo e a maioria das aferências segue os nervos esplâncnicos (parassimpáticos).

\section{Considerações finais}

O centro pontino da micção é o núcleo eferente cerebral final que controla o funcionamento do trato urinário inferior. O PMC sofre fortes influências da PAG que, por sua vez, traz consigo modulações de centros superiores e informações aferentes vesicais. O mecanismo neuronal de switch armazenamento-micção, e fim da micção, permanece largamente desconhecido e ainda requer estudos adicionais. Existem diferentes tipos de descrições neuroanatômicas na literatura sobre conexões aferentes e eferentes, envolvendo estruturas centrais que participariam do controle da micção, contudo, não há evidências de conexões diretas entre o PMC e o PCC, suscitando a dúvida se estes seriam núcleos independentes.

Em centros superiores, o córtex parece ter importância chave na percepção de estímulos provenientes da bexiga, enquanto o giro anterior do cíngulo parece participar das ações voluntárias no controle da micção. O córtex pré-frontal apresenta função crucial na decisão de armazenar e evitar a micção, de acordo com o contexto emocional e social.

Apesar de a importância de áreas prosencefálias, mesencefálicas e pontinas ser relativamente bem descrita na literatura, a importância de estruturas bulbares no controle da micção não é totalmente esclarecido.

O NTS, localizado na porção dorsal do bulbo, constitui o sítio primário no sistema nervoso central para onde se projetam diferentes tipos de aferências somatoviscerais. Porém, ainda permanece incógnito sobre qual seria sua real função sobre o controle vesical. Chen et al. ${ }^{28}$ relataram que estímulos em áreas controladoras da função cardiovascu- 
lar, como a região rostroventrolateral do bulbo e o NTS, produziram alterações na atividade dos nervos pélvicos ${ }^{28}$. Regiões da bexiga inervadas pelos nervos pélvicos sofreram contrações quando estimulados e, inversamente, a inibição nervosa ocasionou relaxamento vesical. Por outro lado, a estimulação da região caudoventrolateral do bulbo, conhecida por exercer ações depressoras no sistema cardiovascular, não produziu alterações na atividade dos nervos pélvicos parassimpáticos e, consequentemente, não causou contração do músculo detrusor.
Kavia, Dasgupta e Fowler' finalizaram seu estudo com uma brilhante frase que ainda é válida: "Algumas questões sobre 'o quê' controla a micção e armazenamento vêm sendo respondidas"”. Todavia, os circuitos envolvidos no controle central da micção ainda apresentam muitos aspectos funcionais não bem compreendidos, e sua elucidação poderia contribuir para o desenvolvimento de novos tipos de terapias para poder serem úteis em condições fisiopatológicas que acometem o controle da micção e armazenamento urinário.

\section{Referências}

1. Kavia RB, Dasgupta R, Fowler CJ. Functional imaging and the central control of the bladder. J Comp Neurol. 2005;493:27-32.

2. Fukuyama H, Matsuzaki S, Ouchi Y, Yamauchi H, Nagahama Y, Kimura J, et al. Neural control of micturition in man examined with single photon emission computed tomography using 99mTc-HMPAO. Neuroreport. 1996;7:3009-12.

3. Barrington F. The relation of the hind-brain to micturition. Brain. 1921;44:23-53.

4. Betts CD, Kapoor R, Fowler CJ. Pontine pathology and micturition dysfunction. Br J Urol. 1992;70:100-2.

5. Komiyama A, Kubota A, Hidai $H$. Urinary retention associated with a unilateral lesion in the dorsolateral tegmentum of the rostral pons. J Neurol Neurosurg Psychiatry. 1998;65:953-4.

6. Renier WO, Gabreels FJM. Evaluation of diagnosis and non-surgical therapy in 24 children with a pontine tumour. Neuropaediatrie. 1980;11:262-73.

7. Blok BF, Holstege G. Direct projections from the periaqueductal gray to the pontine micturition center (M-region). An anterograde and retrograde tracing study in the cat. Neurosci Lett. 1994;166:93-6.

8. Bradley WE, Conway CJ. Bladder representation in the pontinemesencephalic reticular formation. Exp Neurol. 1966;16:237-49.

9. Koshino K. Spontaneous potential activities related to the intravesical pressure in the pontine area of the cat. Jpn J Physiol. 1970;20:272-80

10. Taniguchi N, Miyata M, Yachiku S, Kaneko S, Yamaguchi S, Numata A. A study of micturition inducing sites in the periaqueductal gray of the mesencephalon. J Urol. 2002;168:1626-31.

11. Holstege G. Descending motor pathways and the spinal motor system: Limbic and non-limbic components. Prog Brain Res. 1991;87:307-421.

12. Blok BF, Holstege G. Two pontine micturition centers in the cat are not interconnected directly: Implications for the central organization of micturition. J Comp Neurol. 1999;403:209-18.

13. Redfern WS, Yardley CP. Contribution of the periaqueductal gray matter to the development of hypertension in the spontaneously hypertensive rat. J Auton Nerv Syst. 1990;31:231-9.

14. Rosen SD, Paulesu E, Frith CD, Frackowiak RS, Davies GJ, Jones T, et al. Central nervous pathways mediating angina pectoris. Lancet. 1994;344:147-50.

15. Weiller C, May A, Limmroth V, Juptner M, Kaube H, Schayck RV, et al.
Brain stem activation in spontaneous human migraine attacks. Nat Med 1995;1:658-660.

16. Valet $\mathrm{M}$, Sprenger $\mathrm{T}$, Boecker $\mathrm{H}$, Willoch $\mathrm{F}$, Rummeny $\mathrm{E}$, Conrad B, et al. Distraction modulates connectivity of the cingulo - frontal cortex and the midbrain during pain - an fMRI analysis. Pain. 2004;109:399-408.

17. Mallory BS, Roppolo JR, Groat WC. Pharmacological modulation of the pontine micturition center. Brain Res. 1991;546:310-20.

18. Andrew J, Nathan PW. The cerebral control of micturition. Proc RSM. 1965;58:553-5.

19. Athwal BS, Berkley KJ, Hussain I, Brennan A, Craggs M, Sakakibara R, et al. Brain responses to changes in bladder volume and urge to void in healthy men. Brain. 2001;124:369-77.

20. Zhang H, Reitz A, Kollias S, Summers P, Curt A, Schurch B. An fMRI study of the role of suprapontine brain structures in the voluntary voiding control induced by pelvic floor contraction. Neuroimage. 2005;24:174-80.

21. Sakakibara R, Uchida Y, Uchiyama T, Yamanishi T, Hattori T. Reduced cerebellar vermis activation during urinary storage and micturition in multiple system atrophy: 99mTc-labelled ECD SPECT study. Eur J Neurol. 2004;11:705-8.

22. Andrew J, Nathan PW. Lesions on the anterior frontal lobes and disturbances of micturition and defaecation. Brain. 1964;87:233-62.

23. Dasgupta R, Critchley H, Dolan RJ, Fowler CJ. Changes in brain activity following sacral neuromodulation for urinary retention. J Urol. 2005:174(6):2268-72.

24. Andersson KE, Persson K. The L-arginine/nitric oxide pathway and nonadrenergic, non-cholinergic relaxation of the lower urinary tract. Gen Pharmacol. 1993;24(4):833-9.

25. Groat WC. Anatomy and physiology of the lower urinary tract. Urol Clin North Am. 1993;20(3):383-401.

26. Nishizawa 0, Sugaya K, Noto H, Harada T, Tsuchida S. Pontine micturition center in the dog. J Urol. 1988;140(4):872-4.

27. Yoshimura N, de Groat WC. Neural control of the lower urinary tract. Int $J$ Urol. 1997:4(2):111-25.

28. Chen SY, Chai CY. Coexistence of neuron integrating urinary bladde activity and pelvic nerve activity in the same cardiovascular areas of the pontomedulla in Cats. Chin J Physiol. 2002;45(1):41-5. 